\title{
Confinement and Chiral Symmetry Breaking from an ensemble of interacting Instanton-dyons(monopoles) in SU(2) QCD
}

\author{
Rasmus Larsen ${ }^{1, a}$ \\ ${ }^{1}$ Department of Physics and Astronomy, Stony Brook University, Stony Brook NY 11794-3800, USA
}

\begin{abstract}
We show how the increase in the Instanton-dyon density can explain both Confinement and Chiral symmetry breaking. We simulate an ensemble of 64 interacting Instanton-dyons for 2 colors and 0 or 2 quark flavors. We find that at low temperatures, the high density of dyons prefer a symmetric density, which leads to the confining value of the Polyakov Loop. At the same time the Chiral condensate is highly sensitive to the Polyakov Loop. As the Polyakov Loop gets close to the confining value, the Chiral condensate develops a non-zero expectation value, thus breaking Chiral symmetry.
\end{abstract}

\section{Introduction}

The talk was based on the two papers [1] [2].

We show how a picture of instanton-dyons can lead to the confinement-deconfinement transition for 2 colors and 0 or 2 quark flavors and chiral symmetry breaking for 2 flavors. This is possible due to the dyons being dependent on the Polyakov loop, and at the same time being topological objects, which is needed in order to create the almost-zero-mode zone from the zero-mode solutions around each of the dyons.

The precise model is the interacting instanton-dyon model, where we treat 64 dyons as individual 3 dimensional configurations, interacting at the classical level. The free energy density is found from Monte-Carlo simulations using the Metropolis algorithm. The quantum weight of dyons was calculated by [3].

\section{Dyons}

Instanton-dyons are topological configurations found by [4][5], which can be understood as the building blocks of the finite temperature instanton, the caloron. Since we work with $S U(2)$ we have 2 types of dyons. We call them $M$ and $L$ dyons. The dyons are observed only when one introduces a non-trivial expectation value in some direction of the $A_{4}^{a}$ field. We work with the third color direction $a=3$.

$$
\begin{aligned}
<A_{4}^{3}> & \equiv 2 \pi T v, \\
\text { Polyakov loop } P & =\cos (\pi v)
\end{aligned}
$$

\footnotetext{
a e-mail: Rasmus.N.Larsen@stonybrook.edu
} 
where we call the constant $v$ for holonomy.

The dyons have magnetic and electric charge, and their classical action is linear dependent on the holonomy. We show all of these stats in table 1.

Table 1. Quantum numbers of the four different kinds of the instanton-dyons of the SU(2) gauge theory. The three rows are action times $\frac{g^{2}}{8 \pi^{2}}$ and electric and magnetic charges.

\begin{tabular}{l|c|c|c|r}
\hline & $M$ & $\bar{M}$ & $L$ & $\bar{L}$ \\
\hline$S \frac{g^{2}}{8 \pi^{2}}$ & $v$ & $v$ & $1-v$ & $1-v$ \\
$\mathrm{e}$ & 1 & 1 & -1 & -1 \\
$\mathrm{~m}$ & 1 & -1 & -1 & 1 \\
\hline
\end{tabular}

\section{Interactions}

The simulations done was based on finding the free energy density, such that we could determine the dyon density and Polyakov loop for each explored temperature, and thus the behavior of the vacuum.

The free energy density $f$ is found from the formula [1]

$$
f=\frac{4 \pi^{2}}{3} v^{2} \bar{v}^{2}-2 n_{M} \ln \left[\frac{d_{\nu} e}{n_{M}}\right]-2 n_{L} \ln \left[\frac{d_{\bar{\nu}} e}{n_{L}}\right]+\Delta f
$$

where $\Delta f$ is the correction to the free energy density coming from the interactions. The first term on the right side is the holonomy potential due to the introduction of a non-zero $A_{4}^{a}$ field in the third color direction. The terms in the middle is the entropy of the $M$ and $L$ dyons. The factor of 2 is because we always have the same amount of dyons and anti-dyons. $n_{i}$ is the dyon density and $d_{v}$ is the density of a free gas of dyons. $\bar{v}=1-v$.

There are three different interactions. The classical interactions, the corrections to the metric, and the fermionic interactions.

$$
\Delta f \sim \Delta D_{D D}+V_{D}-N_{f} \log (\operatorname{det}(\hat{\mathbf{T}}))
$$

\subsection{Classical Interactions}

The classical interactions[1] is calculated as 2-point interactions

$$
\Delta D_{D D}=\sum_{j>i} \Delta S_{D_{i} D_{j}}
$$

where $\Delta S_{D_{i} D_{j}}$ is the correction to the action between dyon $\mathrm{i}$ and dyon $\mathrm{j}$. If the two dyons are a dyon and its anti-dyon, we have for distances larger than $x_{0}$

$$
\begin{aligned}
\Delta S_{D \bar{D}} & =-2 \frac{8 \pi^{2} v}{g^{2}}\left(\frac{1}{x}-1.632 e^{-0.704 x}\right) e^{-M_{D} r T} \\
x & =2 \pi v r T
\end{aligned}
$$

For the rest of the combinations we have

$$
\begin{aligned}
\Delta S_{D D} & =\frac{8 \pi^{2} v}{g^{2}}\left(-e_{1} e_{2} \frac{1}{x}+m_{1} m_{2} \frac{1}{x}\right) e^{-M_{D} r T} \\
x & =2 \pi v r T
\end{aligned}
$$


The charges $e_{i}$ and $m_{i}$ are given in table 1 .

For distances smaller than $x_{0}$ we have a core between dyons of same type ie. $L L, M M, \bar{L} \bar{L}, \bar{M} \bar{M}$, $L \bar{L}$ and $M \bar{M}$

$$
\begin{aligned}
\Delta S_{D D} & =\frac{v V_{0}}{1+\exp \left[\sigma\left(x-x_{0}\right)\right]} \\
x & =2 \pi v r T
\end{aligned}
$$

where $x_{0}$ is the size of the dyons core. We worked with $x_{0}=2, V_{0}=20$ and $\sigma=1$.

\subsection{Metric}

The correction to the metric comes from the determinant [1]

$$
\begin{aligned}
G= & \delta_{m n} \delta_{i j}\left(4 \pi v_{m}-2 \sum_{k \neq i} \frac{e^{-M_{D} T\left|x_{i, m}-x_{k, m}\right|}}{T\left|x_{i, m}-x_{k, m}\right|}+2 \sum_{k} \frac{e^{-M_{D} T\left|x_{i, m}-x_{k, p \neq m}\right|}}{T\left|x_{i, m}-x_{k, p \neq m}\right|}\right) \\
& +2 \delta_{m n} \frac{e^{-M_{D} T\left|x_{i, m}-x_{j, n}\right|}}{T\left|x_{i, m}-x_{j, n}\right|}-2 \delta_{m \neq n} \frac{e^{-M_{D} T\left|x_{i, m}-x_{j, n}\right|}}{T\left|x_{i, m}-x_{j, n}\right|}
\end{aligned}
$$

This determinant is not always positive, and we therefore cut it off using the following prescription: If all eigenvalues are positive

$$
\begin{aligned}
& V_{D}=-\log [\operatorname{Det}(G)]: V_{D}<V_{\max } \\
& V_{D}=V_{\max }: V_{D}>V_{\max }
\end{aligned}
$$

and for one or more negative eigenvalues

$$
V_{D}=V_{\max } .
$$

We used $V_{\max }=100$.

\subsection{Fermionic Interactions}

The fermionic interactions comes from the overlap of zero-modes sitting at dyons and anti-dyons of type $L$ only, due to fermions being anti-periodic [2]

$$
T_{i j}=<i|D| j>\sim \exp (-2 \pi(1-v) r / 2)
$$

Expanding in zero-modes sitting at each dyon gives the following formula for the determinant of the Dirac operator

$$
(\operatorname{det}(\hat{\mathbf{T}}))^{N_{f}}
$$

with

$$
\hat{\mathbf{T}} \equiv\left(\begin{array}{cc}
0 & \mathbf{T}_{i j} \\
-\mathbf{T}_{j i} & 0
\end{array}\right)
$$

Where $T_{i j}$ is the correction between dyon $i$ and antidyon $j$. 


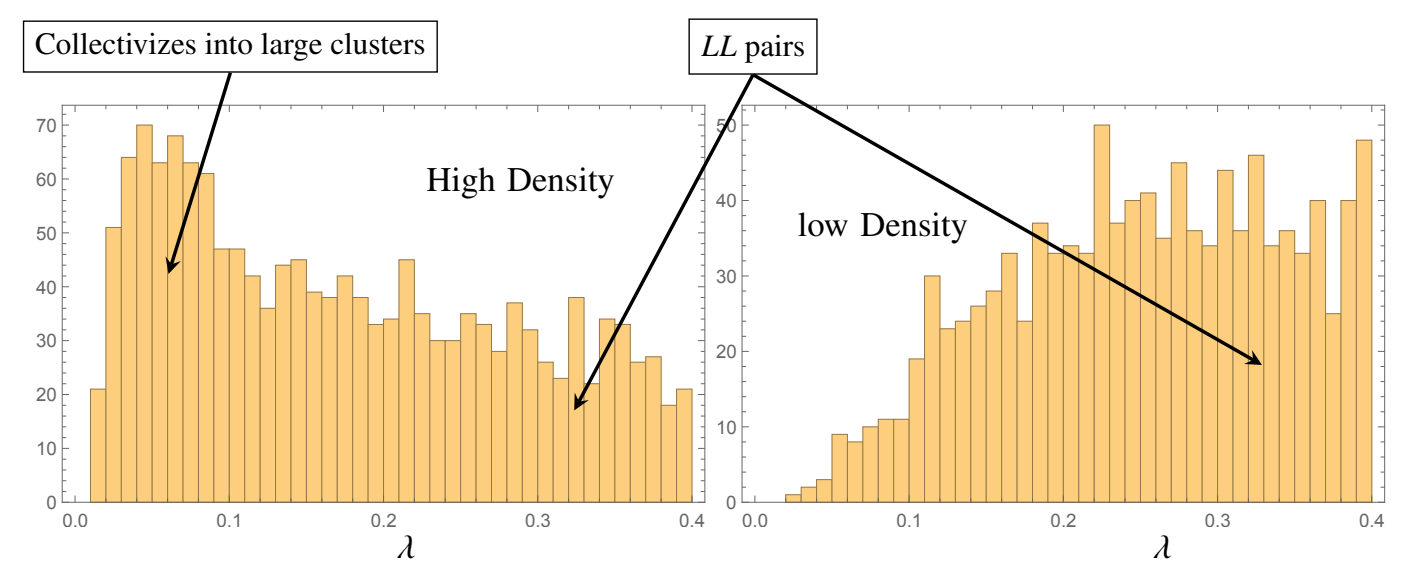

Figure 1. (left) Eigenvalue distribution for $n_{M}=n_{L}=0.47, N_{F}=2$ massless fermions at $S=7$. (right) Eigenvalue distribution for $n_{M}=n_{L}=0.08, N_{F}=2$ massless fermions at $S=7$.

\section{Chiral Condensate}

The chiral condensate is obtained from the almost-zero-mode zone. This is the finite volume equivalent of the Banks-Casher relation

$$
|<\bar{\psi} \psi>|=\pi \rho(\lambda)_{\lambda \rightarrow 0, m \rightarrow 0, V \rightarrow \infty}
$$

The eigenvalues are obtained from the $T_{i j}$ matrix coming from the overlap of the fermionic zeromodes. For low dyon densities (Fig. 1 right) one finds an eigenvalue distribution that is independent of volume. For high dyon densities (Fig. 1 left) one finds an area close to $\lambda=0$ that scales with volume. We therefore fitted this behavior to random matrix theory [6], to obtain the chiral condensate.

The area close to zero was fitted to

$$
\begin{aligned}
\rho(x) & =V \Sigma_{2}\left[\frac{x}{2}\left(J_{2}(x)^{2}-J_{1}(x) J_{3}(x)\right)+\frac{1}{2} J_{2}(x)\left(1-\int_{0}^{x} d t J_{2}(t)\right)\right] \\
x & =\lambda V \Sigma_{1}
\end{aligned}
$$

The two values of $\Sigma_{i}$ was used to extrapolate between two different volumes to decide whether the required volume dependence was present. The extrapolation was done, since in the transition between chiral restored and chiral broken phase, it could be hard to distinguish. An example of this intermediate region is shown in figure 2.

\section{Data}

In order to obtain the free energy we use a standard approach

$$
\begin{aligned}
e^{-F(\lambda)} & =\int D x e^{-\lambda S} \\
F(1) & =\int_{0}^{1}<S(\lambda)>d \lambda+F(0)
\end{aligned}
$$




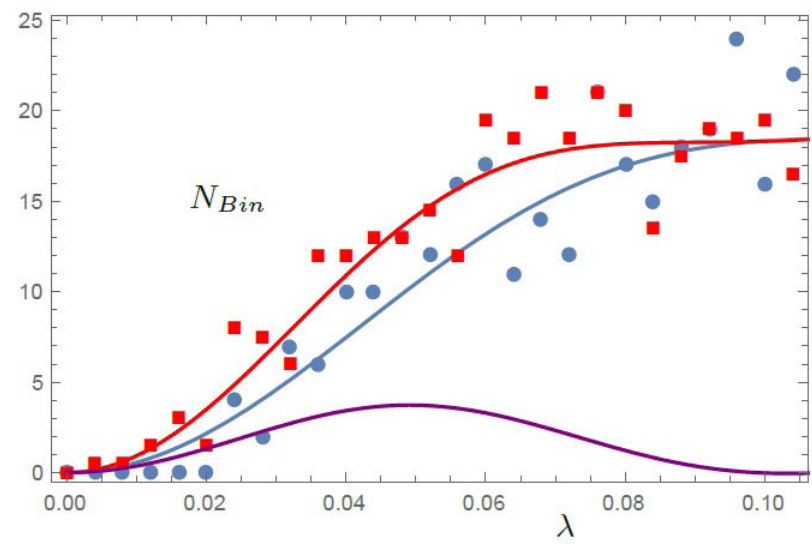

Figure 2. The points are the eigenvalue distribution for 64 (blue circles) and 128 (red squares) dyons at $S=8$ and density of dyons $n_{M}=0.33, n_{L}=0.20, N_{F}=2$. The curves are the fit with eq. (18) with $\Sigma_{2,64}=1.30 \pm 0.06$ and $\Sigma_{2,128}=1.28 \pm 0.06$ and the scaling as $\Sigma_{1,64}=0.79 \pm 0.05$ and $\Sigma_{1,128}=0.51 \pm 0.04$ for these two cases, respectively. The lower purple line is the difference between the two fits. Eq. (18) gives $\Sigma=0.38 \pm 0.13$, while the maximum of the difference between the two curves give $\Sigma=0.3$ after normalizing the difference (note: This approach of using the maximum of the difference between the two volumes, has not been used to analyze the data, but is simple used here to visualize the effect).

one therefore needs to simulate the expectation value of the action $\langle S(\lambda)>$ for different $\lambda$. In our simulations we did it for 20 points, with more points close to $\lambda=0$.

In order to find the dominating configuration at each temperature explored, an initial search was performed in order to find the region of interest. After this a more detailed final run was done. For each combination of parameters, a Monte-Carlo simulation was performed. The following parameters was used:

$\lambda$ : An integration variable used in order to obtain the free energy from the expectation value of the action.

$v$ : The holonomy, related to the Polyakov loop.

$r$ : The radius of the $S_{3}$ sphere, which was the "box" the simulation was done in.

$N_{M}$ : The amount of $M$ dyons in the system.

$M_{d}$ : The cutoff mass, needed since the amount of $M$ and $L$ dyons is not equal.

$S$ : The action of one instanton, is related to the temperature.

The different range of the parameters is shown in table 2 for the 2 flavor case. This gives in total 1440000 configurations.

In order to obtain such a large amount of different configurations, all simulations was done on a NVIDIA gtx980 graphics card, using Cuda code. This meant that several thousand configurations could be run at the same time, reducing simulation time from more than a year, to around a month for one setup, ie. 0 or 2 flavors.

\subsection{Free Energy}

For $n_{M}=n_{L}$ the free energy is symmetric around $v=0.5$ (center symmetry) for 0 flavors (Fig. 3) but not for 2 flavors (Fig. 4). This is due to interactions of fermionic zero-modes only happening between 
Table 2. The input parameters used for the final run for 2 flavors.

\begin{tabular}{l|c|c|r}
\hline & Min & Max & Step size \\
\hline$\lambda$ & 0 & 0.1 & $1 / 90$ \\
$\lambda$ & 0.1 & 1.0 & 0.1 \\
$\nu$ & 0.175 & 0.525 & 0.025 \\
$r$ & 1.05 & 2.00 & 0.05 \\
$N_{M}$ & 16 & 26 & 2 \\
$M_{d}$ & 3 & 6 & 1 \\
$S$ & 5 & 9.5 & 0.5 \\
\hline
\end{tabular}

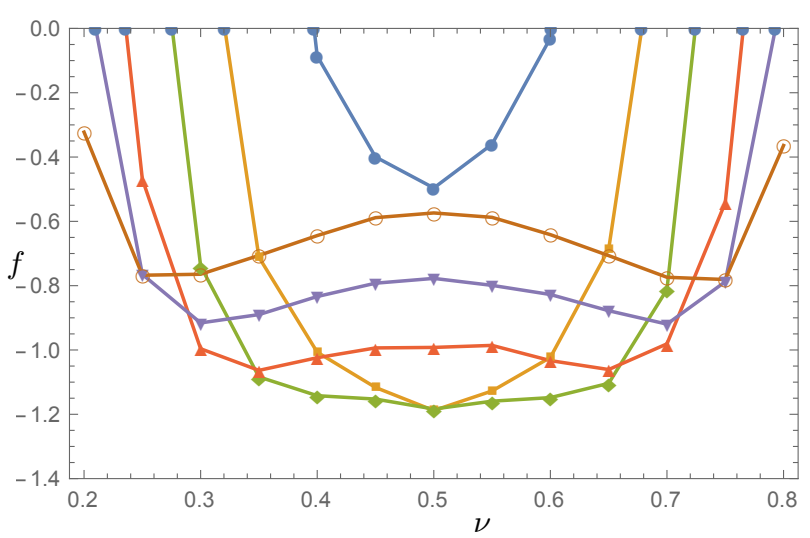

Figure 3. 0 flavors: Free Energy density $f$ as a function of $v$ at $S=6, M_{D}=2$ and $n_{M}=n_{L}$. The different curves corresponds to different densities. $\bullet n=0.53, \mathbf{\square} n=0.37, \diamond n=0.27, \boldsymbol{\Delta} n=0.20, \mathbf{\nabla} n=0.15, \circ n=0.12$. Not all densities are shown.

$L$ type dyons. This means that for 0 flavors, as the temperature increases, the minimum in the middle at $v=0.5$ has to decide on which of the other minimums it goes to, thus spontaneously breaking the symmetry, in a first or second order transition. In the case of 2 flavors, there is only one minimum that moves closer to $v=0.5$ as the temperature decreases.

\section{Numerical results}

\subsection{0 flavors}

The case of no fermions[1] is strongly affected by the breaking of center symmetry. In terms of dyons, breaking of center symmetry is observed as the density of $M$ and $L$ type dyons becoming different. In figure 5 we show how the densities of the dyons and Polyakov loop are highly related. When the densities of $M$ and $L$ dyons become the same, the Polyakov Loop becomes zero, and stays zero. 


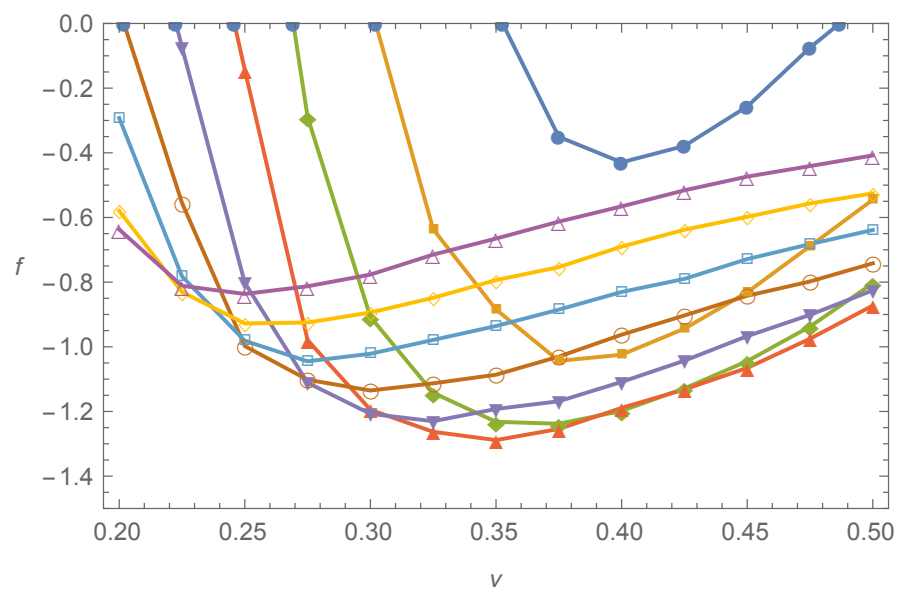

Figure 4. 2 flavors: Free energy density $f$ as a function of the holonomy parameter $v$. Different curves are for different dyon densities. The densities are $(0.47, \bullet),(0.37, \mathbf{\square}),(0.30, \bullet),(0.24, \mathbf{\Delta}),(0.20, \mathbf{v}),(0.16, \circ),(0.14, \square)$, $(0.12, \diamond),(0.10, \Delta)$. Not all densities are shown. $S=8.5$ and $n_{M}=n_{L}$.

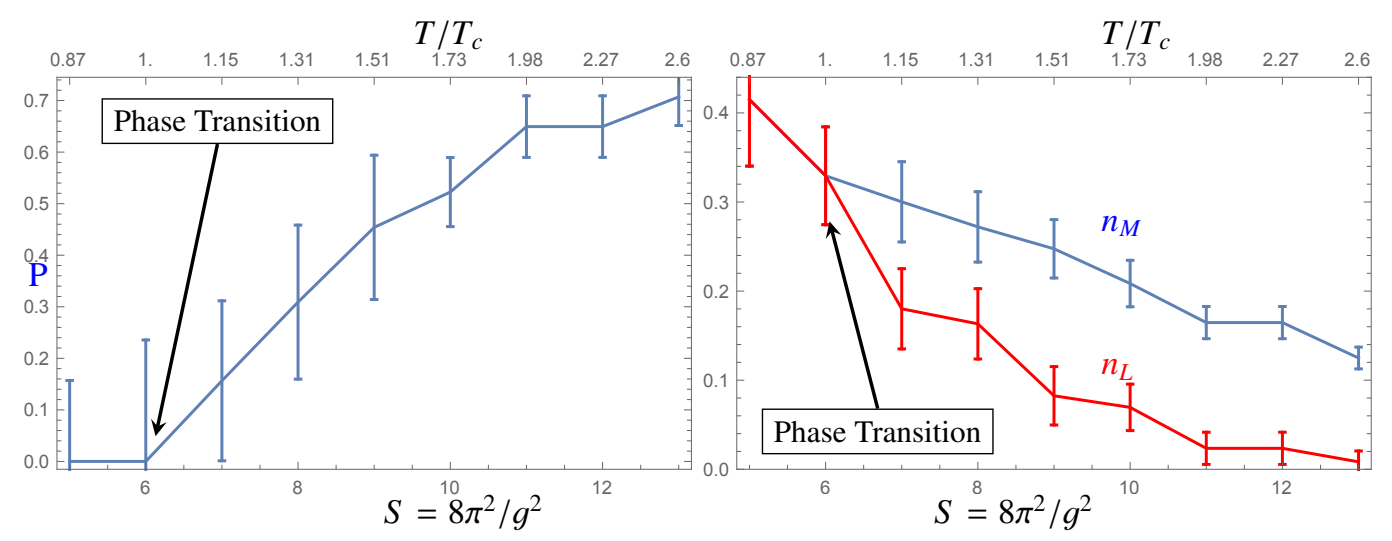

Figure 5. 0 flavors: (left) Polyakov loop $P$ and (right) densities of $M$ and $L$ dyons $n_{i}$ as a function of action of one instanton $\mathrm{S}$ (lower) or temperature $T / T_{c}$ (upper).

\subsection{2 flavors}

The two flavor case is quite different from the quenched case, due to the center symmetry being broken from the beginning. This means that there is no quick transition, as one minimum goes into another minimum. Instead a smooth transition is observed, as the increased density of dyons forces the Polyakov loop closer to zero. We show this in figure 6.

As the temperature decreases, the effective interactions between the zero-modes becomes longer ranged, due to their holonomy dependence. When the density has become large enough, and the 


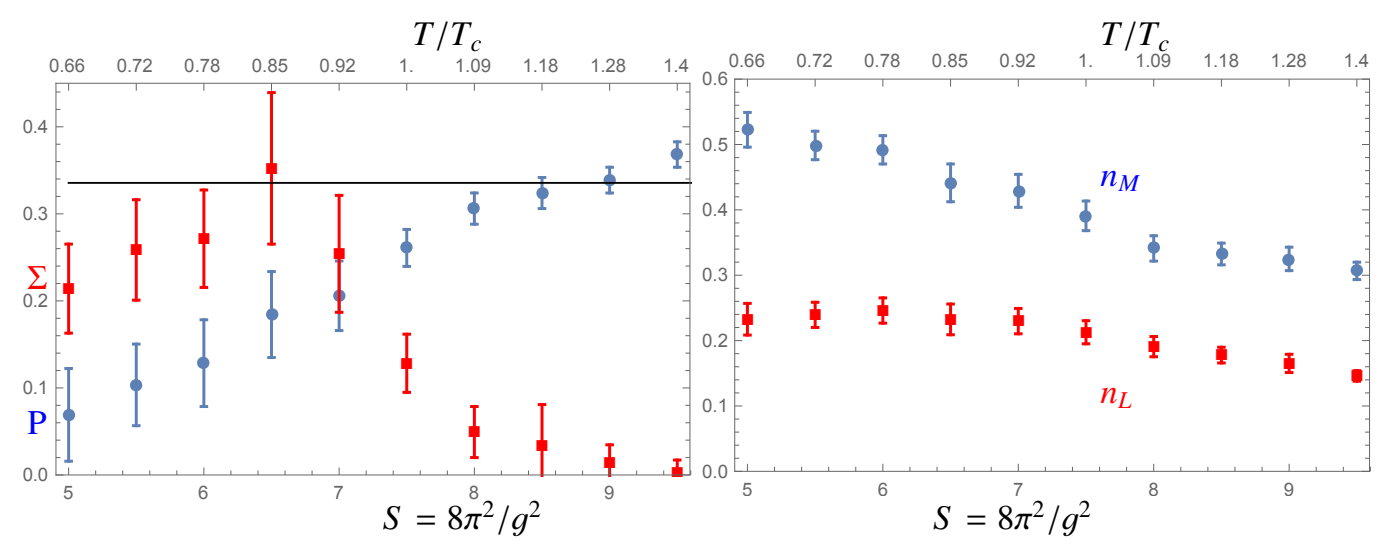

Figure 6. 2 flavors: (left) The Polyakov loop $P$ and the chiral condensate $\Sigma$ and (right) Density of $M$ and $L$ dyons $n_{i}$ as a function of action of one instanton $\mathrm{S}$ (lower) or temperature $T / T_{c}$ (upper).

Polyakov loop small enough, a non-zero chiral condensate is observed. This is also a smooth transition, but much faster than what was observed for the Polyakov loop.

\section{Summary}

We have investigated the finite temperature transition for the Polyakov loop for 2 colors and 0 or 2 quark flavors and the chiral symmetry transition for 2 quark flavors through the chiral condensate.

This was done using the model of interacting dyons, where 64 dyons was put into a "box", from which the free energy density was found as a function of a variety of parameters. From this the dominating configurations was found, and it indeed showed that the instanton-dyons can drive both the confinement-deconfinement transition and the chiral symmetry transition. The results also showed why confinement and chiral symmetry breaking happens at the same temperature. When the Polyakov loop gets closer to zero, it enhances the interactions between the zero-modes. It is these interactions that creates the chiral condensate. Confinement thus enhances chiral symmetry breaking.

\section{Acknowledgments}

The results reported in this talk was done in collaboration with Edward Shuryak.

\section{References}

[1] R. Larsen and E. Shuryak, Interacting ensemble of the instanton-dyons and the deconfinement phase transition in the SU(2) gauge theory, Phys. Rev. D 92, no. 9, 094022 (2015) doi:10.1103/PhysRevD.92.094022 [arXiv:1504.03341 [hep-ph]].

[2] R. Larsen and E. Shuryak, "Instanton-dyon Ensemble with two Dynamical Quarks: the Chiral Symmetry Breaking," Phys. Rev. D 93, no. 5, 054029 (2016) doi:10.1103/PhysRevD.93.054029 [arXiv:1511.02237 [hep-ph]].

[3] D. Diakonov, N. Gromov, V. Petrov and S. Slizovskiy, "Quantum weights of dyons and of instantons with nontrivial holonomy," Phys. Rev. D 70, 036003 (2004) [arXiv:hep-th/0404042]. 
[4] T. C. Kraan and P. van Baal, "Monopole constituents inside SU(n) calorons," Phys. Lett. B 435, 389 (1998) [arXiv:hep-th/9806034].

[5] K. -M. Lee and C. -h. Lu, "SU(2) calorons and magnetic monopoles," Phys. Rev. D 58, 025011 (1998) [hep-th/9802108].

[6] J. J. M. Verbaarschot, "Handbook Article on Applications of Random Matrix Theory to QCD," arXiv:0910.4134 [hep-th]. 